\title{
VOM GEOGRAPHIESTUDIUM AN BRITISCHEN UNIVERSITÄTEN
}

\author{
Walther Manshard
}

\begin{abstract}
Der Geograph, dem es vergönnt war einige Jahre an einer britischen Universität zu studieren oder zu unterrichten, wird mancherlei anregende Beobachtungen mit in die Heimat zurückbringen. In folgenden ist versucht worden, einige Notizen über das Geographiestudium auf den britischen Inseln aufzuzeichnen.

Wenn der englische Student mit 18 Jahren nach Absolvierung seiner Schulzeit oder zwei Jahre später nach der Ableistung seiner Wehrdienstpflicht zur Universität zugelassen wird, hat er häufig schon auf der « Secondary Grammar School » einen spezielleren geographischen Unterricht erhalten. Auf der Oberstufe ( 4 th and 6th Form 》) kann er sich infolge des sehr schmiegsamen Lehrplanes schon früh auf einige Fächer konzentrieren. So sehr natürlich diese gründliche Ausbildung auf den Schulen zu begrüßen ist, so wird diese Entwicklung doch von den Universitäten nicht immer gern gesehen, da die Zahl der übrigen Nebenfächer für das « Higher School Certificate » oder neuerdings das «Certificate in Education 》 beschränkt ist, und häufig die breitere allgemeine Wissensbildung zu kurz kommen läßt. Die Zahl der Studenten, die ganz aus eigenen Mitteln studieren, ist begrenzt. Ein großzügiges Stipendiensystem, das besonders für alle ehemaligen Kriegsteilnehmer organisiert wurde, verhilft den meisten Studierenden, die in ihren schulischen Leistungen über dem Durchschnitt liegen, zu erheblichen Unterstützungen aus öffentlichen Mitteln.
\end{abstract}

Für das Geographiestudium im Hauptfach («Honours Degree») sind in der Regel 3-4 Jahre erforderlich. Dabei muß aber hinzugefügt werden, daß vor der Aufnahme in einen solchen Kurs an verschiedenen Universitäten noch eine Art Vor- oder Zwischenprüfung in mehreren Pflichtfächern abzulegen ist. (Inter B. A., Inter B. Sc., oft z. B. mit Latein in der «Faculty of Arts».) Diese Prüfungen sind als ein wichtiges Gegenmittel der Universität gegen eine zu frühe Spezialisierung gedacht.

Das angelsächsische Universitätssystem unterscheidet sich bekanntlich erheblich von demjenigen kontinentaler Ūniversitäten. Die britischen Universitäten sind unabhängige Körperschaften (die bis 1947 sogar im Parlament vertreten waren) und erst neuerdings größere finanzielle Zuwendungen vom Staat erhalten. Für jedes Studienjahr ist ein bestimmter Lehrplan mit festgelegten Vorlesungen und Übungen vorgeschrieben, an dem der Student teilzunehmen hat. Schriftliche Prüfungen werden am Ende eines Trimesters (《Terminals») und nach Abschluß des akademischen Jahres (《Sessionals») abgehalten. Diese Prüfungen sowie die Besprechungen mit dem «Tutor» (Berater) des Studenten sollen den Lehrkräften einen Ein. blick in den Fortschritt ihrer «Under graduates» ermöglichen. Die Abschlußprüfungen (《Finals»), die zur Erlangung des ersten akademischen Grades (B. A.: Bachelor of Arts, B. Sc.: Bachelor of Science, oder an anderen Universitäten $z$. B. in Schottland zum M. A.: Master of Arts), führen, werden meistens schriftlich durchgeführt. Wie auch im Schulwesen wird mündlichen Prüfungen ( Orals»), - die zwar an einigen. Universitäten stattfinden - keine besondere Bedeutung beigemessen. Zur Wahrung möglichst großer Objektivität im Prüfungswesen erhalten alle Geographiestudenten einer Universität die gleichen Grundfragen, und nur ein Teil der schriftlichen Prüfung berücksichtigt die Wahlgebiete ( Optionals») des Kandidaten. Alle Ergebnisse werden gleichmäßig durchgepunktet, und so ließe sich, wie man gelegentlich scherzhaft bemerkt hat, das gegenwärtige Wissen eines Studenten «auf zwei Stellen nach dem Komma » ausrechnen. Auch jede unabhängige Universität zieht zur Prüfung bekannte Fachvertreter aus benachbarten 
Universitäten als Nebenprüfer und Gutachter heran. Selbständige eigene Arbeiten (kleinere Forschungen, «field work» usw.) werden für die Festsetzung der Klasse des «Degrees» (z. B. I., II., III.-Class) mit eingereicht. Die Berichte des «Tutors » über die wissenschaftliche und persönliche Entwicklung des Studenten versuchen, die etwas unpersönliche Art des Punktsystems zu mildern.

Welche Berufsaussichten eröffnen sich dem jungen ausgebildeten Geographen nach Abschluß seines Examens? Von den Provinzuniversitäten geht die Mehrzahl (über $80 \%$ ) nach der Ableistung eines einjährigen Lehrerbildungskurses an einem "Department of Education“ in das Lehrfach. Außerdem ist allen Geographen die Zulassung zu den Aufnahmeprüfungen des öffentlichen Verwaltungsdienstes („Civil Service“) und des auswärtigen Dienstes („Foreign Service“) möglich. Unter den freien Berufen steht die Wirtschaft an erster Stelle, und viele Volkswirtschafter graduieren (B. Sc. Econ.) mit Wirtschaftsgeographie als Wahlfach. Natürlich vermittelt auch hier das Geographiestudium zunächst nur einen mehr allgemeinen Überblick und der Bildungswert steht durchaus im Vordergrund. Die speziellere Berufsausbildung hat sich dem Studium erst anzuschließen. So beginnt der Vermessungsbeamte des "Colonial Service" oder der zukünftige Landesplaner die eigentliche praktische Fachausbildung erst nach Eriverb des ersten akademischen Grades. Selbstverständlich finden auch eine Anzahl Geographen alljährlich in den Kolonien und den „Dominions des Commonwealth" Anstellungen.

Über den Lehrplan und die geographischen Zielsetzungen der verschiedenen „Departments of Geography “ ist es selbstverständlich schwierig, Verallgemeinerungen zu wagen. Gelegentlich wird der Charakter des Instituts schon durch die Namengebung klar, wie z. B. in Aberystwyth als „Department of Geography and Anthropology“. Besonders vielseitige Möglichkeiten bieten die verschiedenen "Colleges“ der Universität London. Während das „University College“ (H. C. DARBY) vor allem die historische Geographie ptlegt, wächst unter Anleitung von S. W. WooLDRIDGE („King's College") eine Generation von gut ausgebildeten Geomorphologen heran. Für die geographische Ausbildung von Landesplanern sei noch Newcastle (G. H. J. DAYsH), für Wirtschaftsgeographie Liverpool (W. SMITH) erwähnt. Interessant ist auch die Entwicklung in Cambridge, wo die exakte Vermessungsarbeit und systematische Geländeaufnahme („Surveying“, vergl. z. B. die Küstenstudien von J. A. STEers oder die Gletscherforschungen von V. Lew's) im Vordergrund standen. Die Betonung dieser Teilgebiete kann natürlich gelegentlich auf Kosten der Länderkunde vor sich gehen, auf die z. B. das Prüfungssystem der Universität London im Verein mit den ihr angeschlossenen „University Colleges“ mehr Gervicht legt.

Selbstständige wissenschaftliche Leistungen werden nur für den Erwerb der höheren akademischen Grade gefordert (M. A., M. Sc., Ph. D., D. Sc. usw.). Dabei ist zwischen «Thesis》 (selbständige Forschungsleistung) und 《Dissertation》 (mehr referierende Darstellung etwa von Problemen) zu unterscheiden. Eine Veröffentlichung der Arbeiten ist nur in wenigen Fällen möglich, und Exemplare der Schriften sind in den Universitätsbibliotheken einzusehen.

Das Niveau des Ph. D. («Doctor of Philosophy ») liegt im allgemeinen höher als das des deutschen Doktors. Der D. Sc. (《Doctor of Science 》) ist einer der höchsten akademischen Titel, der erst nach vieljährigen Forschungsarbeiten verliehen werden kann. Für die relativ späte Entwicklung der Geographie in Großbritannien ist übrigens bemerkenswert, daß Geographen bisher kaum in die Reihen der «Royal Society» (F. R. S.) eingedrungen sind - einer der höchsten wissenschaftlichen Auszeichnungen in England, deren Mitglieder vor allem aus den Naturwissenschaften kommen. Eine Habilitation in unserem Sinne ist unbekannt. Nach dem Erwerb eines akademischen Titels kann der «Graduate » ohne weiteres, wenn er eine Anstellung an einem «Department of Geography » erhalten hat, Vorlesungen halten. Der allmähliche Aufstieg vom «Assistant Lecturer», «Lecturer 》, «Senior Lecturer », «Reader 》zum Professor hängt von der wissenschaftlichen Leistung und der Zahl der offenen Stellen ab. Die meisten dieser Stellen werden öffentlich ausgeschrieben. Daneben gibt es noch zahlreiche weitere Posten, die entweder der Lehre (《Demonstrator», «Tutor») oder der Forschung ( search Fellow », «Research Assistant») gewidmet sind. Die Zahl der Lehrkräfte ist höher als auf dem Kontinent, und selbst kleine «Departments 》 haben oft fünf und mehr Dozenten.

In der Organisation der britischen Geographie spielen die "Geographischen Gesellschaften“ eine wichtige Rolle. Zunächst hat jede Universität eine "Geographical Society“, die vŏ der Stu- 
dentenschaft 'getragen und finanziert wird. Sie veranstaltet Vorträge und Exkursionen im Rahmen der Universität und ihrer näheren Umgebung. An einigen Universitäten (wie Durham, Oxford und Cambridge) gibt es sogar studentische Forschungsgesellschaften, die kleinere Expeditionen (nach Island, Norwegen usw.) ausrüsten. Größere, überregionale Bedeutung kommt der „Royal Geographical Society" (R.G.S.) in London und der "R. Scottish Geogr. Soc." und deren Veröffentlichungen, dem "Geographical Journal“ und dem „Scottish Geographical Magazine“ zu. Das G.J. bringt seiner alten Tradition und der Mitgliederschaft der R.G.S. entsprechend mit besonderer Vorliebe Feldforschungen, in denen geographische Fachvertreter nicht immer genügend zu Wort kamen, und deren Wert oft mehr in der Erkundung als auf rein wissenschaftlichem Gebiet lag. Aus diesen und anderen Gründen haben sich die britischen Hochschulgeographen im „Institute of British Geographers" (I.B.G.) eine eigene Vereinigung geschaffen. Das „I.B.G.“ hält alljährlich Anfang Januar einen Geographentag ab. Die wichtigsten Vorträge dieses Treffens werden in den "Transactions and Papers“ des „I.B.G.“ veröffentlicht. Die englischen Schulgeographen sind in der "Geographical Association" zusammengeschlossen. Ihr von D. LiNToN redigiertes Organ "Geography" bringt häufig auch wissenschaftliche Arbeiten von Bedeutung. Irland besitzt seit dem letzten Weltkrieg eine eigene geographische Zeitschrift. („Irish Geography“.) Als weitverbreitete populär-geographische Zeitschrift sei noch das "Geographical Magazine" erwähnt, da ein Teil seiner Einkünfte für geographische Forschungsaufgaben bereitgestellt wird.

Die britische Geographie hat sich erst in den letzten Jahrzehnten zur vollen Kraft entfalten können. Ihr Ringen um Anerkennung dauerte länger als in anderen Ländern. Bei Angriffen von Nachbarwissenschaften wird vielfach der Gedanke laut: Die Geographie wäre eigentlich ein „post graduate subject", d. h. ein Fach, dessen Studium erst nach Erwerb von wissenschaftlichen Grundlagen in andern Fächern erfolgreich betrieben werden könne. Zweifellos gibt Vieles in der Entwicklung der britischen Geographie diesen Zweifeln Nahrung. C. A. FishER verlieh dieser Überzeugung 1952 in Aberystwyth beredten Ausdruck: Die „erste Generation“ der britischen Geographie kam (bis in die 20er Jahre) aus Nachbarfächern wie Geologie, Geschichte oder Nationalökonomie. Die Schüler dieser Gruppe konnten sich noch nach dem Vorbild ihrer Lehrer ausrichten. In der "dritten Generation“ fehlt jedoch häufig schon diese breitere Grundlage, und es ergibt sich so die Forderung für die Ausbildung von jungen Geographen, die enge „Mid-of-the-Road Geography“ durch eine bessere Berücksichtigung anderer systematischer Wissenschaftszweige von ihrer Einseitigkeit zu befreien.

Obwohl viele der alten Úniversitäten ängstlich auf die Wahrung ihrer traditionellen Rechte bedacht sind, scheut man doch keineswegs vor neuen kühnen Experimenten zurück, wie es z. B. die Gründung des «University College of North Staffordshire » beweist. Hier wird versucht, einen Ausweg aus dem Spezialistentum unserer Universitätsausbildung $\mathrm{zu}$ finden, und bei der gewählten breiten Grundlage kommt besonders die Geographie zu ihrem Recht.

Trotz wirtschaftlicher Notlage und politischer Schwierigkeiten sind in jüngster Zeit in vielen britischen Kolonien großzügige Universitätsneugründungen erfolgt, in denen auch die Geographie immer ausreichend vertreten ist. Daneben sind die Universitäten der britischen Inseln selbst in vieler Hinsicht Pflanzstätten für die geographische Ausbildung im Rahmen des «Commonwealth of Nations》, und viele bekannte Geographen der «Dominions» erhielten ihre Erziehung in Großbritannien.

\section{LES ÉTUDES GÉOGRAPHIQUES DANS LES UNIVERSITÉS ANGLAISES}

La géographie britannique n'a pu se développer complètement que dans les dernières décennies. Son organisation dans les Universités n'est pas encore achevée. Elle se distingue de celle du continent par le fait que les Universités d'Angleterre sont des corps indépendants, qui ne reçoivent que depuis peu des subsides financiers de l'Etat. Les études géographiques exigent d'ordinaire 3 à 4 ans; elles conduisent à divers grades universitaires. Le géographe peut se consacrer à l'enseignement, aux services de l'Etat (Civil Service), à l'économie privée et au service colonial. Beaucoup de géographes trouvent aussi à s'occuper dans les pays du Commonwealth.

\section{DELLO STUDIO DELLA GEOGRAFIA NELLE UNIVERSITÀ BRITANNICHE}

Solo in questi ultimi decenni la geografia britannica si è potuta sviluppare in tutta la sua pienezza. Ne consegue che anche lo studio si trova in fase di assestamento e di sviluppo. Esso si differenzia da quello sul continente anche perchè le Università rappresentano degli enti indipendenti, che solo in questi ultimi anni hanno usufruito di sussidi statali di qualche entità. Lo studio della geografia, quale materia principale, richiede da tre a quattro anni e conduce a diversi gradi accade- 
mici. Quali possibilità professionali per il geografo entrano in linea di conto l'insegnamento, il servizio statale (Civil Service), la libera economia e il servizio coloniale. Molti geografi trovano un interessante campo di attività negli stati dell'Unione.

\title{
ZUR KOLONISATIONSGESCHICHTE VON NEUGUINEA
}

\section{REISE NACH DEN WISSELMEREN}

\author{
Mit Kartenskizze, 4 Photographien und 2 Zeichnungen
}

Arnold Heim

Die folgende Mitteilung, zuerst geschrieben als Brief im Juni 1939 auf der Rückreise von holländisch West-Neuguinea, mag als ein kleiner Beitrag zur Entdeckungs- und Kolonisationsgeschichte der Papuas im Gebirgsinnern betrachtet werden. Er vermittelt die frischen Eindrücke von einem damals vom weißen Manne jüngst berührten Bergland mit den bis dahin auf der neolithischen Kulturstufe lebenden Bergpapuas.

Mein lang gehegter Wunsch, die Papua Neuguineas kennen zu lernen, konnte auf einer Ferienreise erfüllt werden, dank der Mitwirkung des dänischen Kapitäns C. A. Monsted, den ich in Dobo, dem Hauptort der Aru Inseln (Molukken) kennen lernte, wo er eine Holzindustrie mit Sägerei betrieb. Er selbst interessierte sich für Neuguinea und wurde als Steuermann eines seiner Cutter mein Begleiter und bald mein geschätzter Freund.

Bereits hatte ich als Geologe der Shell und der Standard Petroleum Co. of N. Y. die großen und kleinen Sundainseln jahrelang bereist, vergeblich hoffend, noch von der Zivilisation unberührten „Wilden “ zu begegnen. Auf den abgelegenen Molukken traf ich malaische Mischrassen, meist schon in europäischen Kleidern, partiell zivilisiert und missioniert, mit Schulen, Kirchen oder Moscheen. Die originellen hübschen Häuser waren meist zerfallen und durch öde Giebelhütten ersetzt. Die Frauen scheuten vor dem Fremden. Ursprüngliche Kultur und Fähigkeiten waren in den letzten Dezennien fast verschwunden, Haut- und Geschlechtskrankheiten eingekehrt. In den hintersten Nestern hatten sich chinesische Händler festgesetzt, die Schundwaren gegen Kokosnüsse tauschen, im Geheimen auch Alkohol herstellen und verkaufen. Da ergriff ich eine einzigartige Gelegenheit, noch originelle Zustände in Neuguinea zu suchen, dankbar der NKPM (Standard Oil of New York) für die Gewährung von vier Wochen Urlaub.

Bekanntlich ist Neuguinea nach Grönland die größte Insel und das letzte, noch tcilweise unerforschte tropische Gebirgsland der Erde. Es ist pclitisch dreigeteilt in Niederländisch Neuguinea im Westen, Britisch Papua im Südosten, und die frühere deutsche Kolonie Kaiser-IVilhelms-Land, jetzt australisches Mandatgebiet im Nordosten.

Zwei Jahre vor meiner Reise, im April 1937, überflog der an der E.T.H. in Zürich diplomierte holländische Ingenieur F. J. W IsSEL das bewaldete Gebirge westlich des $5000 \mathrm{~m}$ hohen vergletscherten Karstensz Gebirges und entdeckte auf der Wasserscheide nebst zwei kleineren Seen einen sehr grossen, auf dem er kleine Fischerboote erkannte. Diese Seen wurden die Wisselmeren genannt (Meer bedeutet auf holländisch See). Der große der drei Seen ist der Paniai-See.

Bald nach dieser Entdeckung im September 1937 organsierte der Assistent-Resident Cator auf Ambon im Auftrag der Regierung von Niederl. Indien eine Expedition mit Trägern. Vom UtaFluß an der Südküste ausgehend erreichte sie nach zweieinhalb Wochen harten Ringens durch nassen Bergurwald, über zerschrundene Kalkberge und durch Moraste den großen Paniai-See, dem der wilde Utafluß entspringt. Die Entdeckung war eine Sensation für Niederländisch-Indien. Rasch folgten die Berichte in den Tagesblättern und in der Zeitschrift „Nieuw Guinea“. ${ }^{1}$ Die Wisselmeren wurden, wenn auch etwa $18 \mathrm{~km} \mathrm{zu}$ weit westlich, in den Karten von Neuguinea eingetragen. Sie liegen nordöstlich der Aru-Inseln (Fig. 1).

Mit Erlaubnis der Regierung konnte ich in Dobo, gemeinsam mit Kapitän Monsted, eine kleine Expedition vorbereiten. Wir benützten eines seiner kleineren Segelboote mit eingebautem 12 HP Motor, benannt Spray ${ }^{2}$. I, 1948.

1 Vergl. C.C.F.M. LE Roux: De Bergpapoeas van Nieuw-Guinea en hun Woongebied, Bd.

2 Ihm liegt eine eigenartige Geschichte zugrunde. Es wurde im Jahr 1933 in Bangkok, Siam, von dem Basler Schlosser Niederhauser gebaut, der sich in den Kopf gesetzr hatte, damit allein eine Reise um die Erde zu machen. $\mathrm{Da} ß$ er, mit der Schweizerflagge am Mast, allein mit einer Katze an Bord, über Timor die Aru-Inseln erreichte, ist eine wunderbare Leistung. Dort war er aber erschöpft, hatte nichts mehr zu essen und beendete damit seine Reise. 